\title{
Gamificación en procesos de autoentrenamiento y autoevaluación. Experiencia en la asignatura de Arquitectura de Computadores
}

\author{
M. Espinilla, A. Fernández, J. Santamaría y A. Rivera \\ Departamento de Informática, \\ Universidad de Jaén, España \\ \{mestevez, ahilario, jslopez, arivera\}@ujaen.es
}

\begin{abstract}
Resumen En la educación, la gamificación de procesos está suponiendo una excelente solución para aumentar la motivación del alumnado para desempeñar las actividades de su aprendizaje. Así, en dicho entorno, la gamificación se traduce en brindar a los estudiantes una motivación adicional inmediata que les permita alcanzar una tarea a largo plazo. Nuestro interés en este trabajo se enfoca en compartir la experiencia del uso de dinámicas y mecanismos de juego para incentivar el proceso de aprendizaje autónomo del alumno a través de los procesos de autoentrenamiento y autoevaluación en la preparación de una prueba objetiva para la asignatura de Arquitectura de Computadores en los estudios de Ingeniería en Informática de la Universidad de Jaén.
\end{abstract}

Palabras clave: Gamificación, autoentrenamiento, autoevaluación, aprendizaje autónomo, arquitectura de computadores.

Resumen Gamification is based on the application of concepts and techniques of games in other environments with the aim of encouraging certain behaviors or concrete actions. In education, the gamification is assuming an excellent solution to increase student motivation to perform self-learning activities. Therefore, the gamification translates to provide students with an immediate extra motivation that allows achieving a long-term task. Our interest in this paper focuses on sharing the experience of the use of dynamic and mechanics of games to improve the process of autonomous learning by means of the processes of self-training and self-assessment in the preparation of an choice test in the subject of Computer Architecture at the University of Jaén.

Keywords: Gamification, self-training, self-assessment, autonomous learning, computer architecture.

\section{Introducción}

La gamificación es una de las diez tendencias relacionadas con la tecnología más destacadas a nivel mundial. Consiste en la aplicación de conceptos y técnicas de juegos en otros entornos que previamente no son tan entretenidos o motivadores. El objetivo final consiste en incentivar determinados comportamientos o recompensar acciones 
concretas [1]. La gamificación no se refiere estrictamente a la creación de juegos sino al empleo de mecanismos propios de juegos en entornos y aplicaciones no lúdicas. De este modo, tal y como hemos indicado previamente, se consigue potenciar la motivación, la concentración, el esfuerzo o la fidelización [2].

Entre todas sus posibles aplicaciones, la gamificación está suponiendo una excelente solución en el campo de la educación. Se ha observado que, en efecto, la motivación del alumnado para desempeñar las actividades de aprendizaje se ve beneficiada por el uso de este tipo de técnicas [3]. En dicho entorno la gamificación se traduce en brindar a los estudiantes una motivación adicional inmediata que permite conseguir alcanzar una tarea de aprendizaje a largo plazo. Además, dicho incentivo permite que la realización de una actividad por parte de los alumnos sea más gratificante [4].

El uso de la gamificación está demostrando un gran crecimiento y unos excelentes resultados en el ámbito educativo. El eje vertebrador de este auge se fundamenta en que los alumnos consiguen realizar una actividad por el disfrute del trabajo en sí mismo [5], el cual implica los siguientes beneficios:

- Aumenta la implicación y la motivación del alumno.

- Refuerza el sentido del trabajo desempeñado.

- Establece un compromiso con el logro de los resultados.

- Ofrece un refuerzo positivo.

- Proporciona una retroalimentación inmediata.

Un sencillo proceso de gamificación en el ámbito educativo superior es mostrar un ranking competitivo que permita comprobar a los alumnos cómo están desempeñando sus actividades respecto al resto de compañeros de la clase en una asignatura. El reconocimiento que supone ocupar los primeros puestos dentro del ranking supondría una motivación adicional.

Esta contribución se enfoca a la asignatura de Arquitectura de Computadores que se imparte en los estudios de Ingeniería en Informática y donde parte de ella se evalúa mediante una prueba objetiva de preguntas tipo test. La preparación de pruebas objetivas es vital para superar un cuantioso número de materias en la educación universitaria, ya que hoy en día hablar de evaluación, en muchas ocasiones, implica hablar de pruebas objetivas.

Son muchos los factores que marcan la equidad entre evaluación y pruebas objetivas, entre todos ellos destacan los siguientes:

- Cubrir un área mayor de conocimiento si están bien confeccionadas.

- Propiciar que el alumno se concentre exclusivamente en el contenido de la materia y no en aspectos como la redacción o la ortografía.

- Facilitar la corrección en un tiempo breve, sobre todo en el caso de grandes grupos.

- Eliminar el juicio subjetivo del docente.

Para la superación de una prueba objetiva, como se ha señalado previamente, un factor esencial es una buena preparación mediante la realización de pruebas de este tipo. A medida que el alumnado lleva a cabo una prueba, puede adquirir nuevo conocimiento, ya que se le proporciona una retroalimentación en tiempo real, es decir, lo que se denomina "autoentrenamiento" [6,7]. Conjuntamente, una vez finalizada la prueba, 
el alumno mide el grado de adquisición de sus conocimientos, al que se le denomina "autoevaluación" [8].

Los procesos de autoentrenamiento y autoevaluación forman parte del aprendizaje autónomo del alumno, demandado en la educación universitaria por el actual Espacio Europeo de Educación Superior. Por tanto, una pieza clave en el aprendizaje autónomo es el conjunto de recursos de aprendizaje que el docente pone a disposición del alumnado. Dichos recursos deben de estar correctamente diseñados para que el alumnado pueda aprender de ellos y con ellos, sin la necesidad de la presencia o ayuda del docente [9].

Nuestro interés en este trabajo se enfoca en compartir la experiencia del uso de dinámicas y mecanismos de juego para incentivar el proceso de aprendizaje autónomo del alumno, a través de los procesos de autoentrenamiento y autoevaluación, y a la hora de preparar la prueba objetiva de la asignatura de Arquitectura de Computadores en los estudios de Ingeniería en Informática de la Universidad de Jaén. Además se presenta la opinión de los alumnos sobre este proceso que ha sido recabada a través de una encuesta de satisfacción. Los comentarios vertidos muestran una opinión, en general, muy favorable al uso de técnicas y dinámicas de juegos en la asignaturas con algunos puntos a mejorar que también son señalados.

Para llevar a cabo este trabajo, en primer lugar se describe el contexto de la asignatura donde se ha llevado a cabo la experiencia. Posteriormente, se describen las técnicas de gamificación que se han utilizado para los procesos de autoentrenamiento y autoevaluación junto con una evaluación por parte del alumnado implicado en la experiencia. Finalmente, se incluye una sección con las conclusiones y los trabajos futuros.

\section{Las asignatura de Arquitectura de Computadores en la Universidad de Jaén}

La asignatura donde se ha llevado a cabo la experiencia en el uso de técnicas de gamificación es la asignatura de Arquitectura de Computadores del Grado de Ingeniería en Informática impartida en la Escuela Politécnica Superior de Jaén en la Universidad de Jaén.

Dicha asignatura, junto a la asignatura de Programación y Administración de Redes, conforma la materia de Arquitectura de Computadores y Redes, la cual es común a la rama de informática. Arquitectura de Computadores se imparte en primer lugar, estando ubicada en tercer cuatrimestre, es decir, en el primer cuatrimestre del segundo curso. Mientras, la asignatura de Programación y Administración de redes se imparte en el cuarto cuatrimestre, es decir, en el segundo cuatrimestre del segundo curso. La carga total lectiva de la asignatura de Arquitectura de Computadores es de 6 créditos ECTS, distribuidos en 2,5 créditos ECTS para clases expositivas, 3 créditos ECTS para clases en pequeño grupo y, finalmente, 0,5 créditos ECTS para tutorías colectivas.

La importancia de la asignatura en el contexto de la titulación viene determinada por el deber de un graduado en Ingeniería en Informática de conocer los diferentes elementos de un computador, así como las distintas arquitecturas avanzadas que actualmente se utilizan. De este modo, se dota al graduado en Ingeniería en Informática de un punto de vista práctico esencial, que le permite conocer a fondo las características 
y el funcionamiento del hardware, saber cómo mejorar el rendimiento de un sistema, y saber comparar dos arquitecturas distintas con el fin de identificar y escoger la mejor en función de las necesidades.

Las competencias de la asignatura de Arquitectura de Computadores son las siguientes:

- Conocimiento y aplicación de las características, funcionalidades y estructura de los Sistemas Distribuidos, las Redes de Computadores e Internet, y diseño e implementación de aplicaciones basadas en ellas.

- Conocimiento y aplicación de los principios fundamentales y técnicas básicas de la programación paralela, concurrente, distribuida y de tiempo real.

- Conocimiento, administración y mantenimiento de sistemas, servicios y aplicaciones informáticas.

- Capacidad de conocer, comprender y evaluar la estructura y arquitectura de los computadores, así como los componentes básicos que los conforman.

Así, los resultados de aprendizaje de la asignatura son los siguientes:

- Conocer y aplicar las características, funcionalidades y estructura de los Sistemas Distribuidos, las Redes de Computadores e Internet. Diseñar e implementar aplicaciones basadas en ellas.

- Conocer y aplicar los principios fundamentales y técnicas básicas de la programación paralela, concurrente, distribuida y de tiempo real.

- Ser capaz de conocer, administrar y mantener sistemas, servicios y aplicaciones informáticas.

- Conocer, comprender y evaluar la estructura y arquitectura de los computadores, así como los componentes básicos que los conforman.

Los contenidos básicos de la asignatura versan sobre la clasificación de arquitecturas paralelas, la evaluación de prestaciones, paralelismo a nivel de instrucción (procesadores segmentados y superescalares), computadores paralelos (multiprocesadores, multicores, procesadores multihebra y multicomputadores), optimización de código y, finalmente, programación paralela.

Respecto a los contenidos teóricos, en los cuales se ha llevado a cabo la experiencia del uso técnicas de gamificación, están desglosados en los siguientes módulos:

- Módulo 1: Introducción.

- Módulo 2: Segmentación de cauce.

- Módulo 3: Procesadores superescalares.

- Módulo 4: Procesadores VLIW.

- Módulo 5: Procesadores vectoriales.

- Módulo 6: Computadores paralelos, programación paralela, prestaciones y redes de interconexión.

Sobre las metodologías y actividades que son llevadas a cabo en la asignatura se incluyen: 
- Clases magistrales expositivas donde se intercalan exposiciones de teoría y ejemplos generales de los contenidos de la asignatura. Además, se incluyen en las clases magistrales expositivas en gran grupo otras metodologías como debates y resolución de ejercicios.

- Clases en pequeño grupo realizadas en laboratorios y en aulas de informática del departamento de informática de la Universidad de Jaén donde se llevan a cabo resoluciones de ejercicios sobre los contenidos teóricos y actividades prácticas con las aplicaciones informáticas.

- Tutorías colectivas consistentes en la aclaración de dudas sobre los contenidos de la asignatura, tanto teóricos como prácticos, en las que se fomenta el debate entre los asistentes para la aclaración de las dudas, bajo la dirección del profesor.

La evaluación de la asignatura se sustenta en el siguiente sistema consistente en tres pilares:

- Dominio de los conocimientos teóricos y operativos de la materia, el cual es evaluado mediante un examen teórico consistente en una prueba objetiva de tipo test, cuestiones cortas teórico-prácticas y la resolución de ejercicios.

- Dominio sobre un tema relacionado con arquitectura de computadores, el cual es evaluado mediante la elaboración de un informe y su defensa.

- Dominio de los conocimientos prácticos de la materia, el cual es evaluado a través de la elaboración de guiones consistentes en cuestiones prácticas, desarrollo de programas, informe de resultados y su posterior defensa.

En esta contribución se expone el uso de técnicas de gamificación para incentivar la preparación de la prueba objetiva de la asignatura a través del proceso de aprendizaje autónomo del alumno mediante los procesos de autoentrenamiento y autoevaluación.

\section{Gamificación en procesos de autoentrenamiento y autoevaluación. Experiencia en la asignatura de arquitectura de computadores}

Durante el curso 2015/2016 en la asignatura de Arquitectura de Computadores de la Universidad de Jaén se han utilizado técnicas y dinámicas de juegos enfocadas a incentivar al alumnado en la preparación de la prueba objetiva del examen teórico.

En esta sección, se describe la experiencia. Para ello, primero se describe el análisis de recursos TIC que se ha realizado para llevar a cabo el proceso de gamificación (Sección 3.1). A continuación, se indican las técnicas que se han utilizado, las cuales hemos denominado recurso gamificador (Sección 3.2). Por último, se indica la evaluación del recurso gamificador por parte del alumnado implicado en la experiencia (Sección 3.3).

\subsection{Elección del recurso TIC para llevar a cabo la experiencia}

Al inicio de la experiencia, se realizó un estudio de las técnicas y dinámicas de gamificación más adecuadas en la educación superior, llevándose a cabo un análisis de plataformas y recursos de gamificación existentes. 
En dicho análisis se estudió tanto plataformas gamificadoras existentes en la red como la posibilidad de adaptar las técnicas y dinámicas de gamificación a la plataforma de docencia virtual que brinda la Universidad de Jaén, ILIAS ${ }^{1}$.

Sobre las plataformas analizadas, se estudiaron con mayor profundidad las siguientes, debido a su relevancia:

- Gameonlab ${ }^{2}$, el cual contiene un conjunto de servicios sobre cómo utilizar el pensamiento de juego para aumentar la participación y compromiso en una organización, pudiéndose adaptar al proceso de autoevaluación y autoentrenamiento de un alumno.

- Socrative ${ }^{3}$, se trata de un sistema de respuesta inteligente con el que el profesor puede lanzar preguntas, quizzes y juegos, a los que los alumnos pueden responder en tiempo real desde sus dispositivos, ya que funciona desde un móvil, desde una tableta, desde un PC o un portátil con conexión a Internet.

- Captainup ${ }^{4}$, se utiliza con el objetivo de aprender sobre gamificación jugando. Este sitio permite tener una experiencia de gamificación desde el punto de vista del individuo gamificado.

- Openbadges ${ }^{5}$, es una iniciativa gratuita de la Fundación Mozilla. Su propósito es reconocer mediante insignias o medallas digitales los aprendizajes adquiridos por una persona en distintos momentos y situaciones tanto formales como informales.

Fruto de dicho análisis se detectó que la capacidad de exportación de preguntas tipo de test desde la plataforma que brinda la Universidad de Jaén, ILIAS, a las plataformas de gamificación existentes era muy limitada, siendo necesario crear nuevamente la base de datos de preguntas tipo de test en la plataforma de destino de gamificación, en el caso de que existiese esa posibilidad.

Debido a este gran obstáculo, se decidió adaptar los recursos de los que dispone ILIAS para la integración de técnicas y dinámicas de gamificación en la asignatura. Este hecho, además, brinda la posibilidad de integrar en un único espacio todos los materiales de la asignatura.

Es de destacar que la revisión realizada sobre las plataformas estudiadas nos permitió adquirir conocimientos sobre cómo aplicar las técnicas y dinámicas de gamificación en los procesos docentes de la asignatura.

\subsection{Recurso Gamificador. Técnicas y mecanismos de dinámicas de juegos}

Para llevar a cabo la experiencia, se creo un espacio denominado recurso gamificador integrado en la plataforma de docencia virtual ILIAS, la cual implementa las técnicas y dinámicas de gamificación.

La gamificación persigue el concepto de refuerzo positivo e implica que a partir de las reacciones a un determinado comportamiento, aumente la posibilidad de que el

\footnotetext{
${ }^{1} \mathrm{http}: / / \mathrm{www} . \mathrm{ilias} . \mathrm{de}$

$2 \mathrm{http}: / /$ www.gameonlab.es/canvas/

${ }^{3} \mathrm{http} / / / \mathrm{www}$. socrative.com/

${ }^{4}$ https://captainup.com/

${ }^{5} \mathrm{http}$ ://openbadges.org/
} 
comportamiento vuelva a ocurrir en el futuro. Para ello, en el recurso gamificador se han creado objetivos y recompensas personalizadas que implican que dicho comportamiento sea la motivación para alcanzar el objetivo.

En nuestro caso, el recurso gamificador cuenta con pruebas tipo test que se realizan a través de la plataforma y se nutren de un repositorio de preguntas tipo test de cada uno de los módulos que integran la asignatura. Dicho repositorio está creado desde el curso académico 2010/2011, actualizándose con nuevas preguntas cada curso académico.

El recurso gamificador con las técnicas y dinámicas de gamificación consiste en la realización de pruebas tipo test a través de la plataforma ILIAS a lo largo de la impartición de la asignatura, utilizando la base datos de preguntas, la cual está desglosada por módulos. Dicho recurso está basado en un sistema de puntuación, mecanismo muy popular y habitual, donde la participación o la finalización adecuada de pruebas tipo test implica obtener puntos, bonificaciones y reconocimientos en el escalafón de la asignatura. Para ello, de forma periódica en la asignatura se han llevado a cabo pruebas de tipo test competitivas que tiene una puntuación de 20 puntos cada una. La puntuación total obtenida puede verse incrementada por diferentes bonificaciones.

Para lograr el proceso de autoentrenamiento y autoevaluación, durante un intervalo de tiempo anterior a la prueba competitiva, están disponibles para el alumnado las pruebas tipo test en la plataforma ILIAS con el objetivo de que los alumnos puedan realizar dichas pruebas tantas veces como deseen, favoreciendo el proceso de autoentrenamiento. Dichas pruebas no puntúan y serán generadas aleatoriamente a partir del repositorio de preguntas tipo test contenido en el espacio virtual de la asignatura en ILIAS. Una vez finalizada cada una de las pruebas de entrenamiento, al alumno se le muestra la respuesta que ha seleccionado en cada pregunta y la respuesta correcta con el fin de que pueda realizar su autoevaluación.

En las pruebas competitivas de tipo test, se aplican logros y recompensas que consisten en mecanismos de juego en los que si se alcanza cierto nivel se pueden obtener bonificaciones que se traducen en la acumulación de puntos extra. En nuestro caso, los logros y recompensas que se establecen son los siguientes:

- Los alumnos que obtengan la mayor puntuación en la prueba competitiva obtendrán 200 puntos, a repartir entre los alumnos que obtengan dicha puntuación.

- Los alumnos que obtengan una puntuación mayor que 5 puntos en la prueba competitiva, obtendrán una puntuación extra de 15 puntos.

- Los alumnos que obtengan una puntuación mayor que 7 puntos en la prueba competitiva, obtendrán una puntuación extra de 30 puntos.

- Los alumnos que obtengan una puntuación mayor que 9 puntos en la prueba competitiva, obtendrán una puntuación extra de 50 puntos.

Respecto a las puntuaciones finales, los alumnos que hayan obtenido una puntuación media de las pruebas en los siguientes intervalos recibirán las siguientes bonificaciones, siempre que aprueben todas las pruebas competitivas:

- [5,7): bonificación de 100 puntos.

- [7,9): bonificación de 200 puntos.

- $[9,10)$ : bonificación de 300 puntos.

- 10: bonificación de 400 puntos. 
Después de cada prueba competitiva, se muestra una clasificación que promueve la competición entre los alumnos para incentivar su participación donde siempre se cuente con la satisfacción de verse en la zona alta de una tabla o, en el caso opuesto, evitar el último puesto de la tabla. Así, al finalizar cada prueba se publica dentro del recurso gamificador en ILIAS un archivo con el ranking de participantes con la puntuación obtenida en la última prueba competitiva realizada, la puntuación global (suma acumulativa de las diferentes pruebas competitivas más las bonificaciones que haya podido lograr), junto con la media de puntuaciones de las pruebas competitivas.

Con el objetivo de incentivar aún más el proceso de autoentrenamiento y autoevaluación, se establecieron como premios dispositivos electrónicos a los alumnos que obtuvieran una mayor puntuación final en la asignatura.

Además, se planificó sortear premios de consolación a los alumnos que completen todas las pruebas tipo test y la encuesta de evaluación del recurso gamificador. De este modo, los alumnos que ocupan la zona baja del ranking no se ven desmotivados al tener un aliciente adicional para continuar con el proceso de autoentrenamiento y autoevaluación. Dichos premios fueron financiados a través del proyecto de innovación docente de la Universidad de Jaén titulado "Gamificación para incentivar los procesos de auto-entrenamiento y auto-evaluación”.

Cabe notar que el recurso gamificador está desarrollado únicamente para llevar a cabo los procesos de autoentrenamiento y autoevaluación, por lo que las pruebas tipo test competitivas realizadas en la plataforma no puntúan para la evaluación de la asignatura. Como se ha comentado, el recurso gamificador está enfocado para motivar el proceso de aprendizaje autónomo del alumno.

Para finalizar, se indica la distribución temporal de las pruebas tipo test competitivas que se llevaron a cabo. Cada una de las pruebas se realizó al menos una semana después de impartir el módulo correspondiente en las clases magistrales expositivas. Las pruebas se realizaron siempre a las 22 horas con el fin de que las pruebas no se solaparan con docencia reglada del grado de Ingeniería Informática, ya que el turno de tarde en la Universidad de Jaén finaliza a las 21:30 horas. Las pruebas se llevaron a cabo en los siguientes días:

- Módulo 1: 5 de octubre de 2015

- Módulo 2: 18 de noviembre de 2015

- Módulo 3 y 4: 15 de diciembre de 2015

- Módulo 5 y 6: 21 de diciembre de 2015

\subsection{Evaluación del recurso gamificador por parte del alumnado}

Al finalizar todos las pruebas tipo test competitivas, se llevó a cabo una encuesta de satisfacción consistente en 8 preguntas donde cada alumno debía indicar su grado de acuerdo o desacuerdo en la siguiente escala: Totalmente en desacuerdo-(1), Desacuerdo-(2), Ni acuerdo ni desacuerdo-(3), De acuerdo-(4) y Totalmente de acuerdo-(5).

Las 8 preguntas contenidas en la encuesta fueron las siguientes:

1. La gamificación te ha servido para comprender la materia.

2. La gamificación te ha servido para preparar la prueba objetiva del examen de teoría. 
3. La gamificación te ha servido para aumentar el interés por la materia.

4. La existencia de premios consistentes en dispositivos electrónicos en la gamificación ha motivado tu participación en las pruebas competitivas.

5. La existencia de un ranking entre compañeros ha motivado tu participación.

6. La gamificación es un buen recurso didáctico.

7. La distribución de preguntas por módulos te ha parecido adecuada

8. La hora de las pruebas competitivas te ha parecido apropiada.

La encuesta fue completada por un total de 40 alumnos, obteniéndose los resultados de la Tabla 1.

Tabla 1. Resultados de la encuesta de satisfacción

\begin{tabular}{|c|c|c|c|c|c|c|}
\hline Pregunta & 1 & 2 & 3 & 4 & 5 & Mediana \\
\hline \hline 1 & $\mathrm{n}=0(0,00 \%)$ & $\mathrm{n}=0(0,00 \%)$ & $\mathrm{n}=4(9,76 \%)$ & $\mathrm{n}=28(68,29 \%)$ & $\mathrm{n}=9(21,95 \%)$ & 4 \\
\hline 2 & $\mathrm{n}=0(0,00 \%)$ & $\mathrm{n}=0(0,00 \%)$ & $\mathrm{n}=5(12,50 \%)$ & $\mathrm{n}=22(55,00 \%)$ & $\mathrm{n}=13(32,50 \%)$ & 4 \\
\hline 3 & $\mathrm{n}=0(0,00 \%)$ & $\mathrm{n}=5(12,50 \%)$ & $\mathrm{n}=14(35,00 \%)$ & $\mathrm{n}=13(32,50 \%)$ & $\mathrm{n}=8(20,00 \%)$ & 3 \\
\hline 4 & $\mathrm{n}=2(5,00 \%)$ & $\mathrm{n}=3(7,50 \%)$ & $\mathrm{n}=10(25,00 \%)$ & $\mathrm{n}=11(27,50 \%)$ & $\mathrm{n}=14(35,00 \%)$ & 4 \\
\hline 5 & $\mathrm{n}=0(0,00 \%)$ & $\mathrm{n}=3(7,50 \%)$ & $\mathrm{n}=9(22,50 \%)$ & $\mathrm{n}=16(40,00 \%)$ & $\mathrm{n}=12(30,00 \%)$ & 4 \\
\hline 6 & $\mathrm{n}=1(2,50 \%)$ & $\mathrm{n}=0(0,00 \%)$ & $\mathrm{n}=3(7,50 \%)$ & $\mathrm{n}=15(37,50 \%)$ & $\mathrm{n}=21(52,50 \%)$ & 5 \\
\hline 7 & $\mathrm{n}=1(2,50 \%)$ & $\mathrm{n}=1(2,50 \%)$ & $\mathrm{n}=7(17,50 \%)$ & $\mathrm{n}=17(42,50 \%)$ & $\mathrm{n}=14(35,00 \%)$ & 4 \\
\hline 8 & $\mathrm{n}=10(25,00 \%)$ & $\mathrm{n}=7(17,50 \%)$ & $\mathrm{n}=5(12,50 \%)$ & $\mathrm{n}=4(10,00 \%)$ & $\mathrm{n}=14(35,00 \%)$ & 3 \\
\hline
\end{tabular}

Además, en la encuesta a los alumnos se les brindó la posibilidad de realizar observaciones sobre la experiencia en el uso de técnicas de gamificación. Entre los comentarios vertidos, destacan los siguientes comentarios positivos de los alumnos sobre la experiencia:

- Ayuda realmente a llevar al día la asignatura, sobre todo en los aspectos teóricos de la misma, pues en la gamificación también se fomenta la competitividad entre los compañeros de clase motivando al alumnado para realizarla.

- La gamificación sí sirve para preparar el examen de teoría, pues en cierta medida te obliga a estudiar periódicamente el temario a modo de evaluación continua.

- Daros a los docentes de la asignatura la enhorabuena por la iniciativa a pesar de que haya que seguir puliendo dicha idea.

- Me parece una iniciativa genial y nos prepara bastante para el examen final viendo preguntas tipo sobre la materia.

- Me ha parecido bien organizado y bastante útil.

- Los pruebas competitivas han ayudado a ir estudiando día a día para poder participar en la competición. Es una muy buena forma de hacer que llevemos la asignatura más al día.

- Ha estado muy guay competir con los compañeros.

Entre los puntos a mejorar señalados por el alumnado destaca la hora de realización de la prueba tipo test competitiva que puntúa para el ranking, ya que se ha manifestado 
un desacuerdo sobre la hora para llevar a cabo dicha prueba. En próximos cursos, a inicio de curso se consensuará con los alumnos la hora para llevar a cabo la prueba competitiva que puntúa para el ranking.

Otros comentarios relativos a la gamificación que podrán ser tenidos en cuenta en los próximos cursos académicos son los siguientes:

- Debería facilitarse un listado con las preguntas del repositorio para facilitar el estudio y así no tener que acceder a ILIAS.

- Cada prueba competitiva debería versar sobre un único módulo de teoría.

- Se deberían incluir más preguntas tipo test en cada prueba.

Como se ha comentado previamente, para incentivar aún más el proceso de autoentrenamiento y autoevaluación, se establecieron como premios dispositivos electrónicos a los alumnos que obtuvieran una mayor puntuación final en las pruebas competitivas, sorteándose, además, premios de consolación a los alumnos que habían llevado a cabo todas las pruebas tipo test competitivas y que habían completado la encuesta de satisfacción del recurso gamificador.

La Figura 1 muestra una fotografía del día que se hizo entrega de los premios a los galardonados y a los alumnos ganadores en el sorteo.

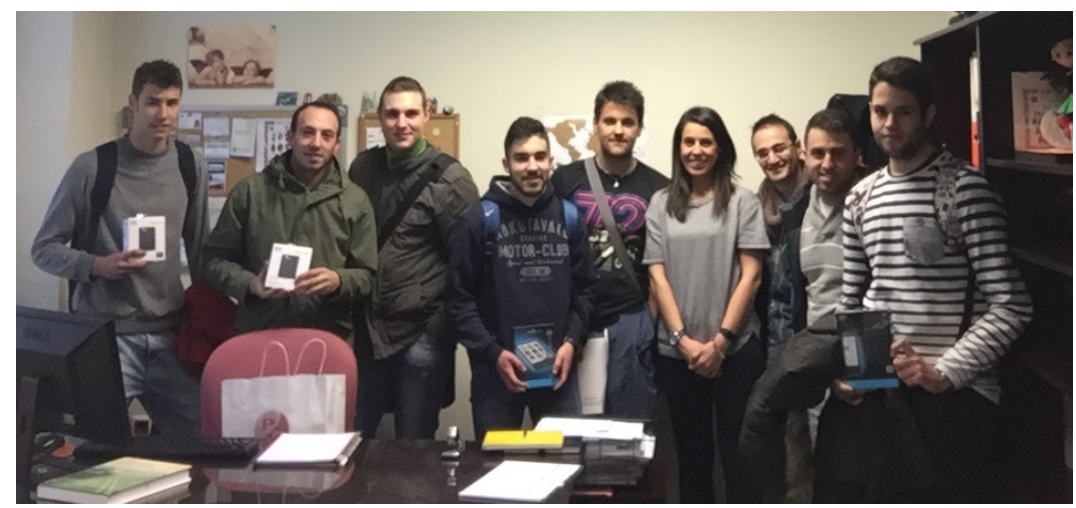

Figura 1. Alumnos galardonados en la gamificación con la profesora del grupo de teoría de la mañana.

\section{Conclusiones y trabajos futuros}

En esta contribución hemos presentado la experiencia de utilización de técnicas de gamificación en el entorno académico universitario. El fin último fue aumentar la motivación del alumnado para realizar el proceso de autoentrenamiento y autoevaluación para la preparación de la prueba objetiva en la asignatura de Arquitectura de Computadores del grado de Ingeniería en Informática de la Universidad de Jaén. 
Durante el primer cuatrimestre del curso académico 2015/2016 se llevó a cabo con éxito la puesta en funcionamiento de las técnicas de gamificación. La evaluación de la utilización de técnicas de gamificación por parte del alumnado que ha cursado la asignatura ha sido muy satisfactoria. Nuestros trabajos futuros se encaminan a la incorporación de nuevas técnicas para aumentar el nivel de competitividad, considerando además la retroalimentación recibida por el alumnado durante el curso académico en que se puso en marcha la prueba piloto.

\section{Agradecimientos}

Esta publicación ha sido financiada por el proyecto de innovación docente de la Universidad de Jaén con código PID24_201416 titulado "Gamificación para incentivar los procesos de auto-entrenamiento y auto-evaluación".

\section{Referencias}

1. S. Deterding, D. Dixon, R. Khaled, and L. Nacke, "From game design elements to gamefulness: Defining gamification," pp. 9-15, 2011.

2. S. Deterding, K. O’Hara, M. Sicart, D. Dixon, and L. Nacke, "Gamification: Using game design elements in non-gaming contexts," pp. 2425-2428, 2011.

3. D. Dicheva, C. Dichev, G. Agre, and G. Angelova, "Gamification in education: A systematic mapping study," Educational Technology and Society, vol. 18, no. 3, pp. 75-88, 2015.

4. C. Nevin, A. Westfall, J. Martin Rodriguez, D. Dempsey, A. Cherrington, B. Roy, M. Patel, and J. Willig, "Gamification as a tool for enhancing graduate medical education," Postgraduate Medical Journal, vol. 90, no. 1070, pp. 685-693, 2014.

5. L. Moccozet, C. Tardy, W. Opprecht, and M. Leonard, "Gamification-based assessment of group work," pp. 171-179, 2013.

6. E. Martin, "Conclusiones: Un currículo para desarrollar la autonomía del estudiante," La universidad ante la nueva cultura educativa. Enseñar y aprender para la autonomía, pp. 285292, 2003.

7. J. Pozo and C. Monereo, "Introducción. un currículo para aprender. profesores, alumnos y contenidos ante el aprendizaje estratégico," El aprendizaje estratégico. Enseñar a aprender desde el currículo, pp. 11-25, 2002.

8. A. Garcia-Beltran, R. Martinez, J. Jaen, and S. Tapia, "Self-assessment in virtual teaching and learning environments," Revista de Educación a Distancia, 2006.

9. G. McNamara and J. O'Hara, "The importance of the concept of self-evaluation in the changing landscape of education policy," Studies in Educational Evaluation, vol. 34, no. 3, pp. 173-179, 2008. 